\title{
Unsteady 1-D Thrust Modeling with EOS Effects for Ram Accelerator Experiments at Different Bores
}

\author{
C. Knowlen, ${ }^{a}$ P. Bauer, ${ }^{b}$ T. Bengherbia, ${ }^{b}$ Y.F. Yao, ${ }^{c}$ A.P. Bruckner, ${ }^{a}$ and M. Giraud ${ }^{d}$ \\ ${ }^{\text {a }}$ University of Washington, Seattle, WA 98195, WA, USA \\ ${ }^{\mathrm{b}}$ Laboratory PPRIME, ENSMA, Futuroscope, 86960, France \\ ${ }^{c}$ University of the West of England, Bristol BS16 1QY, UK \\ d Exobal Consulting, Saint-Louis la Chaussée, 68300, France
}

\begin{abstract}
Advances made to the unsteady, one-dimensional (1-D) modeling of the thermally choked ram accelerator thrust-Mach number characteristics include the use of real-gas equations of state to account for the compressibility effects of the combustion products. Equations of state based on generalized empirical and theoretical considerations are incorporated into a 1-D computer code to predict the combustion end state equilibrium conditions when the propellant starts out a relatively high fill pressure $(>2.5 \mathrm{MPa})$ and the projectile acceleration exceeds $100 \mathrm{~km} / \mathrm{s} 2$. The objective of this work is to improve the unsteady 1-D model as a useful tool to predict the thrust of the thermally choked ram accelerator propulsive mode by utilizing key results from the more computationally intensive 2-D or 3-D simulations. New thrustMach number calculations compared with experimental data from 25-mm, 30-mm, 38-mm, 90-mm, and 120-mm-bore experiments are generally in good agreement until the point where enhanced accelerations are observed, presumably due to projectile material combustion. The results of this investigation indicate the need for more research on ram accelerator flow fields and the role projectile material may play in the combustion process.
\end{abstract}

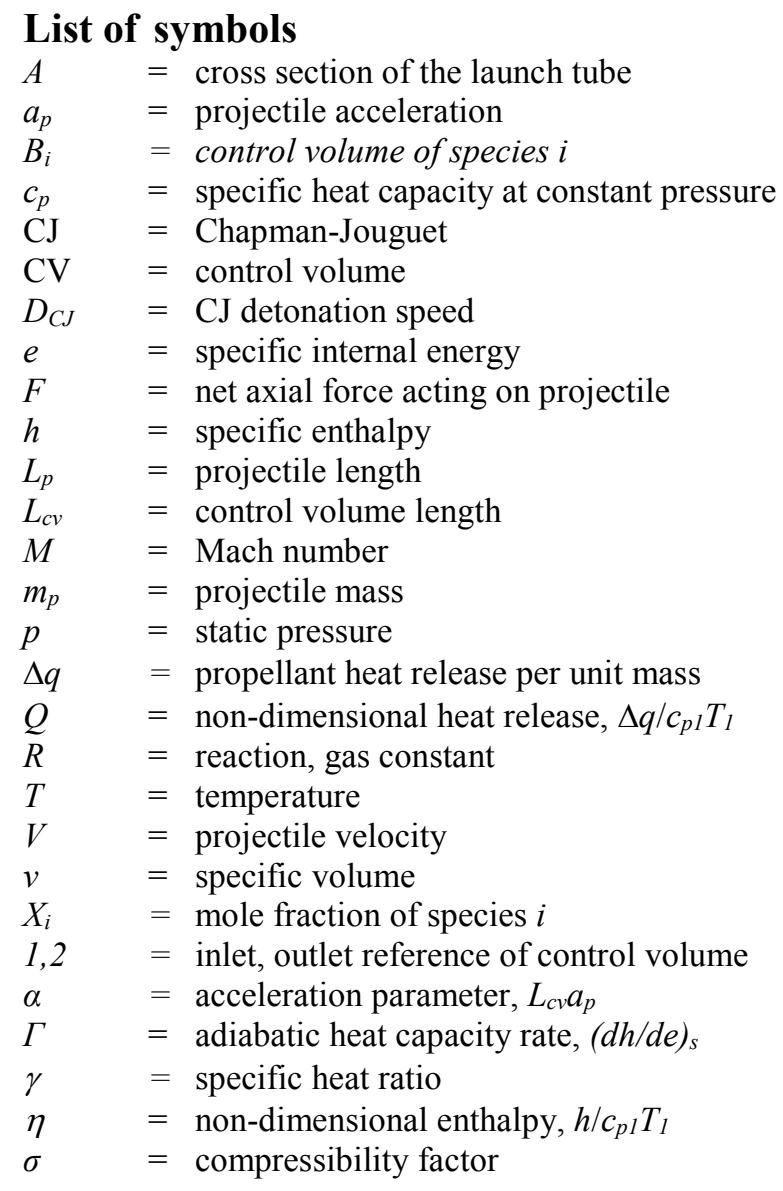

Corresponding author: bauer@ensma.fr 


\section{Introduction}

A propulsion concept known as the ram accelerator [1], or RAMAC, based on an in-tube ramjet propulsion cycle, has successfully been used to accelerate projectiles up to velocities of nearly $3 \mathrm{~km} / \mathrm{s}$ in a tube filled with gaseous propellant (e.g., $\mathrm{CH}_{4}+\mathrm{Air}$ ). Many experimental investigations on the RAMAC concept have been carried out at laboratories around the world, such as University of Washington (UW) 38-mm-bore facility at propellant fill pressures up to $20 \mathrm{MPa}[2,3,4]$; FrenchGerman Research Institute Saint Louis (ISL) 30-mm-bore and 90-mm-bore devices at fill pressures up to 4.5 MPa [5]; US Army Research Laboratory (ARL) 120-mm-bore device at fill pressures up to 8.5 MPa [6]; and Tohoku University (TU) 25-mm-bore device at fill pressures up to $3.5 \mathrm{MPa}$ [7]. Corresponding computational fluid dynamic (CFD) studies have also been carried out at other institutions such as Universitie of Poiteirs, France [8,9,10], Seoul National University, Korea [11], National Research Laboratory, USA [12], and ARL [13].

Projectile acceleration and flow field characteristics of RAMAC operation in various velocity regimes are dependent on the projectile velocity $(V)$ relative to the Chapman-Jouguet $(\mathrm{CJ})$ detonation speed $D_{C J}$ of the propellant [3]. In the subdetonative velocity regime $\left(V<D_{C J}\right)$, RAMAC thrust-Mach number characteristics are readily modelled by assuming a subsonic combustion zone that is terminated by thermal choking behind the projectile, as shown in Fig. 1. In this propulsive mode, thrust generated by the high projectile base pressure results from a normal shock system stabilized on the projectile body by thermal choking of the flow at the full tube area $[1,2]$. Experiments show that this propulsive mode operates effectively in the Mach number range of $3-4.5$. The refinements to the theoretical thrust-Mach number calculations of the RAMAC presented here only apply during operation in the subdetonative velocity regime, thus the higher velocity RAMAC propulsive modes [3] were not considered.

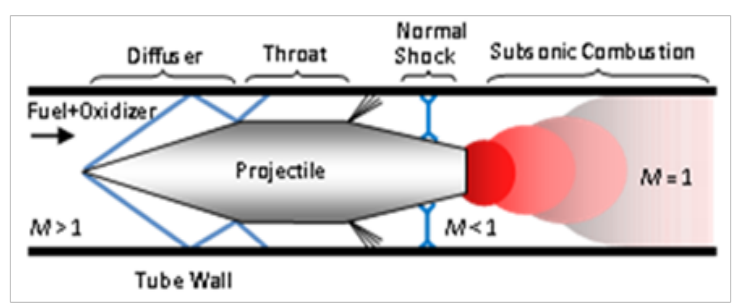

Figure 1. Flow field schematic of the thermally choked ram accelerator propulsive mode.

In prior studies using a chemical equilibrium code named TARAM for calculating the unsteady thrust of the thermally choked RAMAC propulsive mode, good agreement between 1-D unsteady theory and 38-mm-bore RAMAC experiments was attained [14,15]. Including real-gas equations of state (EoS) (e.g.; Boltzmann, Redlich-Kwong, Percus-Yevick, Becker-Kistiakowsky-Wilson (BKW)) to account for compressibility effects extends the applicability of the TARAM code to better predict RAMAC thrust when the fill pressure is increased beyond $3 \mathrm{MPa}[16]$.

In this paper, the efficacy of this unsteady, real-gas 1-D computational model using the Boltzmann and BKW EoS is investigated by comparing thrust predictions with representative experimental data from the 25-mm, 38-mm, 90-mm, and 120-mm-bore RAMAC facilities of TU, UW, ISL, and ARL, respectively. These experiments utilized similar projectile geometries, propellants (CH4, O2, N2), fill pressures (3-5 MPa), and velocity range $(1.1-1.8 \mathrm{~km} / \mathrm{s})$ as listed in Table 1 (Section 4). The projectile materials (Al, Mg, Ti), however, differed in these experiments. The theoretical development of the 1-D model used by the TARAM code is presented, the impact of combustion zone length vs. bore dimension is discussed, and comparisons of its thrust predictions with experimental results are evaluated.

\section{One-Dimensional Model}

The quasi-steady one-dimensional thrust-Mach number model for the thermally choked RAMAC propulsive mode was originally developed at the University of Washington [1,2]. In the projectile 
reference frame (see Fig. 2), this model assumes steady flow enters the control volume at supersonic velocity (denoted as state 1) and exits at sonic velocity (denoted as state 2), where it has attained chemical equilibrium while conserving mass and energy. The sum of the streamwise momentum and pressure forces between the incoming and the outgoing flows is the net axial force (thrust) applied to the projectile. The thrust-Mach number characteristics calculated in this manner compare very well with experiments when the rate of acceleration and the fill pressure are below $100 \mathrm{~km} / \mathrm{s}^{2}$ and $2 \mathrm{MPa}$, respectively $[1,2]$.

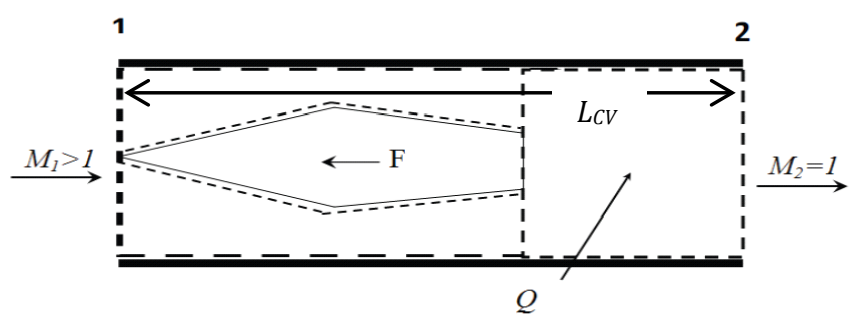

Figure 2. Control volume for 1-D model of the thermally choked RAMAC propulsive mode.

At initial pressures greater than $2.5 \mathrm{MPa}$, the inclusion of real-gas EoS resulted in better agreement between the quasi-steady thrust calculations and experimental results [17,18]. For situations of very high projectile acceleration $\left(>100 \mathrm{~km} / \mathrm{s}^{2}\right)$, a revised unsteady model that includes the effects of a real-gas EoS for the combustion products was developed $[4,19]$. This model accounts for the effect of rapid projectile velocity change on the net thrust by incorporating variations in combustion zone length, $L_{C V}$, and mass of propellant within the control volume. In the reference frame of the projectile, the unsteady mass, energy, and momentum conservation equations are applied to the propellant flow entering and leaving the control volume (Fig. 2); where the conditions at the control volume entrance and the exit planes are identified by subscripts " 1 " and " 2 ," respectively. The influence of combustion heat release on the rate of change of axial streamwise thrust is determined by the introduction of a non-dimensional chemical heat release parameter, $Q=\Delta q / c_{p l} T_{1}$ and a nondimensional thrust parameter, $F / p_{1} A_{1}$, acting on the control volume, where $F$ is equal to the thrust predicted by this RAMAC model.

Analysis of the conservative equations yields a readily applicable set of equations in the form expressed by Bundy et al. [4] and Bauer et al. [19]. After some algebraic manipulation of these relationships, while specifying the end state to be thermally choked; i.e., $M_{2}^{2}=\Gamma_{2} R_{2} T_{2}=1$, and introducing a real-gas EoS, namely, $p v / R T=\sigma(v, T)$, the following expressions are derived:

$$
\begin{aligned}
& \frac{T_{2}}{T_{1}}=\frac{c_{p 1}}{c_{p 2}} \frac{\left(\eta_{1}+\frac{\gamma_{1} R_{1} M_{1}^{2}}{2 c_{p 1}}+Q\right)-\frac{7}{2} \frac{\alpha}{c_{p 1} T_{1}}}{\left(\eta_{2}+\frac{\Gamma_{2} R_{2}}{2 c_{p 2}}\right)\left(1-\frac{\alpha}{\gamma_{1} R_{1} M_{1}^{2} T_{1}}\right)} \\
& \frac{p_{2}}{p_{1}}=\frac{\sigma_{2}}{\sigma_{1}}\left(M_{1} \sqrt{\frac{\gamma_{1} R_{2}}{\Gamma_{2} R_{1}}}-\frac{\alpha}{R_{1} M_{1} T_{1}} \sqrt{\frac{R_{2}}{R_{1} \gamma_{1} \Gamma_{2}}}\right) \sqrt{\frac{T_{2}}{T_{1}}} \\
& \frac{F}{p_{1} A_{1}}=\frac{\alpha}{R_{1} T_{1}}+\frac{p_{2}}{p_{1}}\left(1+\frac{\Gamma_{2}}{\sigma_{2}}\right)-\left(1+M_{1}^{2} \frac{\gamma_{1}}{\sigma_{1}}\right)
\end{aligned}
$$

with: 
$\left.\alpha=L_{C V} a_{p} \quad ; \quad \Gamma=\frac{\partial h}{\partial e}\right)_{S}$

Unlike in the quasi-steady-state formulation of the thermally choked RAMAC thrust $[1,2]$, the preceding equations show that the non-dimensional thrust, $F / p_{1} A_{1}$, is a direct function of both the length of the control volume, $L_{C V}$, and the projectile acceleration, $a_{p}$. An iterative procedure was used to solve for the value of $\alpha$ in Eq. (3), which is an acceleration parameter relating the combustion zone length and projectile acceleration as a function of Mach number for an arbitrarily chosen value for $L_{C V}$ [19]. Based on experimental observations of the luminosity of the flow in 38-mm-bore experiments [2], a value of $L_{C V}=2 L_{P}$ was initially chosen (i.e. $306 \mathrm{~mm}$ in this instance). It was later found that the unsteady thrust predictions were highly dependent on the assumption of $L_{C V}$ dimensions [19]; thus more accurate combustion zone length estimates were needed.

Previous CFD studies, utilizing both axisymmetric and 3-D simulations, addressed laminar and turbulent flows $[8,9]$. These CFD simulations provided detailed flow field information arising from projectile-tube interactions and their influence on the thrust. The calculated peak amplitudes of pressure and temperature and the location of the shock-wave system were validated against available test data [9]. Re-plotted from Bengherbia et al. [10] in Fig. 3 is the corresponding length of the combustion zone determined from these CFD simulations as a function of Mach number, for a 38mm-bore RAMAC with a 153-mm-long projectile. The length of the combustion zone was determined from the projectile base to the point where the area-averaged axial Mach number equals unity. The $L_{C V}$ values plotted in Fig. 3, determined by adding the length of projectile to the combustion zone length (as shown in Fig. 2), are varied from 160 to $560 \mathrm{~mm}$ over the Mach range of 2.9 to 5.1, respectively.

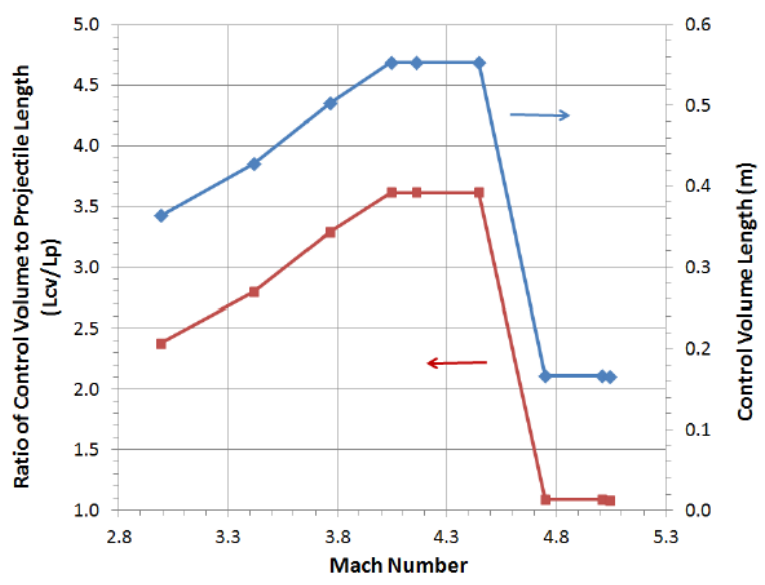

Figure 3. Control volume length from tip of projectile to exit plane where area-average axial Mach $=1[10]$.

In prior studies using the TARAM code for calculating the unsteady thrust of the thermally choked RAMAC propulsive mode, utilizing the $L_{C V}$ based on Mach number dependent nondimensional combustor length (normalized by projectile length, Fig. 3) resulted in good agreement between 1-D unsteady theory and 38-mm-bore RAMAC experiments [14,15]. When comparing experimental data from RAMACs having different bore sizes and geometrically scaled projectiles, however, the appropriate $L_{C V}$ does not necessarily scale with $L_{p}$. Thus, in the results presented here, the combustion zone length is assumed to be fixed at $350 \mathrm{~mm}$, which corresponds to its value for operation at $M_{l}=3.8$ in the $38-\mathrm{mm}$-bore RAMAC. Fixing this length is consistent with assuming that the residence times of the fluid elements are comparable for all bore dimensions in the combustion zone behind projectile, and that the small variations in $\mathrm{CH}_{4} / \mathrm{O}_{2} / \mathrm{N}_{2}$ propellant mole ratios in the experiments are not significant. These assumptions ultimately need verification with appropriate CFDmodeling, a task that has not yet been undertaken. 


\section{Available Equations of State for Combustion Products}

RAMAC operation at fill pressures in the range of $7 \mathrm{MPa}$ to $30 \mathrm{MPa}$ are of interest for launching massive payloads $(>1000 \mathrm{~kg})$ at modest accelerations $\left(<50 \mathrm{~km} / \mathrm{s}^{2}\right)[20,21]$ and achieving very high accelerations comparable with military weapons $\left(>200 \mathrm{~km} / \mathrm{s}^{2}\right)$. Thus it is of interest to develop the ability to incorporate equations of state (EoS) that are appropriate for the RAMAC operation at these elevated fill pressures. Numerous EoS based on generalized empirical and theoretical considerations have been developed by Heuzé [22]. Only the general forms of each EoS incorporated in the TARAM computer program that were used in the present study are presented here.

Several EoS are suited to predict the thermo-chemical properties of combustion products at pressures corresponding to the end state of the RAMAC, which can range from $50 \mathrm{MPa}$ to $1000 \mathrm{MPa}$. A virial type, namely the Boltzmann EoS, has been extensively used and its applicability to the RAMAC calculations for a 38-mm-bore has been widely demonstrated $[4,17,23]$. Nevertheless, the use of another EoS that is appropriate for the pressure range under consideration here, such as the Becker, Kistiakowsky and Wilson (BKW) EoS, is worth investigating for different bore dimensions. The main reason is its applicability to a wide range of temperatures and pressures of combustion products that cover the whole field of gaseous to condensed explosives, based on the appropriate choice of its adjustable parameters.

\subsection{Boltzmann}

The Boltzmann EoS [24] adequately predicts the Chapman-Jouguet properties when the pressure of combustion products does not exceed $200 \mathrm{MPa}$ [25]. This equation of state treats the individual molecules as hard spheres and the mixing rule accounts only for interactions of similar species. The Boltzmann expansion for the compressibility factor is computed by the formula below:

$$
\sigma=1+x+0.625 x^{2}+0.287 x^{3}+0.193 x^{4}
$$

where $x$ is defined as:

$$
x=\sum_{i} \frac{X_{i} B_{i}}{v_{i}}
$$

\subsection{Becker-Kistiakowsky-Wilson (BKW)}

This EoS was introduced in 1921 by Becker, and later modified by Kistiakowsky and Wilson [26]. It can be presented as follows:

$$
\frac{p v}{R T}=1+x e^{\beta x}
$$

with:

$$
x=\frac{\kappa B}{V(T+\theta)^{\alpha}}
$$

and:

$$
B=\sum_{i} x_{i} b_{i}
$$

where $\alpha, \theta, \kappa$ are semi-empirical constants that must be adjusted. In particular, $b_{i}$ are the specific covolumes defined for this EoS. This formulation of the BKW EoS is mostly used for condensed explosives; however, previous research by Heuzé [22] and Bengherbia et al. [14] showed that it could be applied to the calculation of gaseous detonation characteristics at extremely elevated pressures, if all the adjustable parameters were appropriately set. 


\section{Results and Discussion}

The one-dimensional TARAM code for unsteady RAMAC thrust calculations was applied here using the ideal gas, Boltzmann, and BKW EoS respectively; whose adjustable parameter values were validated by comparison with experimental CJ speeds $[27,28]$. The TARAM code is able to calculate the real-gas effects for each EoS by changing the way of the parameter $\sigma$ is computed for both the combustion products and reactants. In order to investigate the applicability of these EoS for predicting the thrust of the RAMAC thermally choked propulsive mode, the present study is aimed at comparing both the calculated velocity profiles and non-dimensional thrust with experimental results from facilities having bore dimensions ranging from $25 \mathrm{~mm}$ to $120 \mathrm{~mm}$, as listed in Table 1 . All experimental parameters needed to predict the thrust-Mach number profiles of the experiments are listed here with the assumption that the reactant temperatures were all $300 \mathrm{~K}$ and the combustion zone length was $350 \mathrm{~mm}$ regardless of bore dimension. Details of the facilities and the results of these particular experiments can be found in cited references $[5,7,13,29]$.

Table 1 Experimental test data matrix from different RAMAC facilities.

\begin{tabular}{|c|c|c|c|c|c|c|c|}
\hline $\begin{array}{c}\text { Facilit } \\
\mathrm{y}\end{array}$ & $\begin{array}{c}\text { Bore } \\
(\mathrm{mm} \\
)\end{array}$ & $\begin{array}{c}\text { Propellant } \\
(\mathrm{moles})\end{array}$ & $\begin{array}{c}\text { Pressure } \\
(\mathrm{MPa})\end{array}$ & $\begin{array}{c}\text { Velocity } \\
\text { Range }(\mathrm{km} / \mathrm{s})\end{array}$ & $\begin{array}{c}\text { Projectile } \\
\text { Mass }(\mathrm{kg})\end{array}$ & $\begin{array}{c}L_{p} \\
(\mathrm{~mm})\end{array}$ & $\begin{array}{c}L_{C V} \\
(\mathrm{~mm})\end{array}$ \\
\hline $\mathrm{TU}$ & 25 & $2.8 \mathrm{CH}_{4}+2 \mathrm{O}_{2}+5.7 \mathrm{~N}_{2}$ & 3.5 & $1.23-1.92$ & 0.022 & 101 & 451 \\
\hline $\mathrm{UW}$ & 38 & $3.0 \mathrm{CH}_{4}+2 \mathrm{O}_{2}+5.7 \mathrm{~N}_{2}$ & 5.1 & $1.15-1.86$ & 0.109 & 153 & 503 \\
\hline $\mathrm{ISL}$ & 90 & $3.0 \mathrm{CH}_{4}+2 \mathrm{O}_{2}+9.9 \mathrm{~N}_{2}$ & 4.0 & $1.33-1.67$ & 1.332 & 396 & 746 \\
\hline ARL & 120 & $3.0 \mathrm{CH}_{4}+2 \mathrm{O}_{2}+10 \mathrm{~N}_{2}$ & 5.2 & $1.25-1.46$ & 4.332 & 522 & 872 \\
\hline
\end{tabular}

\subsection{Velocity-Distance}

Experimental velocity-distance data from these four facilities are plotted in Fig. 4 along with the corresponding 1-D modeling results using the Boltzmann and the BKW EoS with the $L_{C V}$ for each data set listed in Table 1. The theoretical velocity-distance plots are adjusted to intersect the first data point from each experiment to properly represent the performance expectations of the thermally choked RAMAC propulsive mode. The deviation between Boltzmann and BKW EoS velocity-distance calculations is most pronounced in the higher fill pressure cases of the UW and ARL data, which is consistent with the observation that BKW EoS predictions for $D_{C J}$ increase with increasing fill pressure at a greater rate than $D_{C J}$ predictions based on Boltzmann EoS.

It is evident that the Boltzmann predictions agree with experiments better than BKW for the data from UW and ARL plotted here, and the BKW EoS is in better agreement for the TU and ISL data. Since the BKW calculations seem to favor lower fill pressure conditions at two very different projectile size scales ( $25 \mathrm{~mm}$ and $90 \mathrm{~mm}$ RAMACs), this implies that it is the better choice for EoS at fill pressures below $4 \mathrm{MPa}$. Conversely, the Boltzmann EoS predictions are in very good agreement with the experiments at fill pressures greater than $5 \mathrm{MPa}$, regardless of bore dimension and projectile length. 

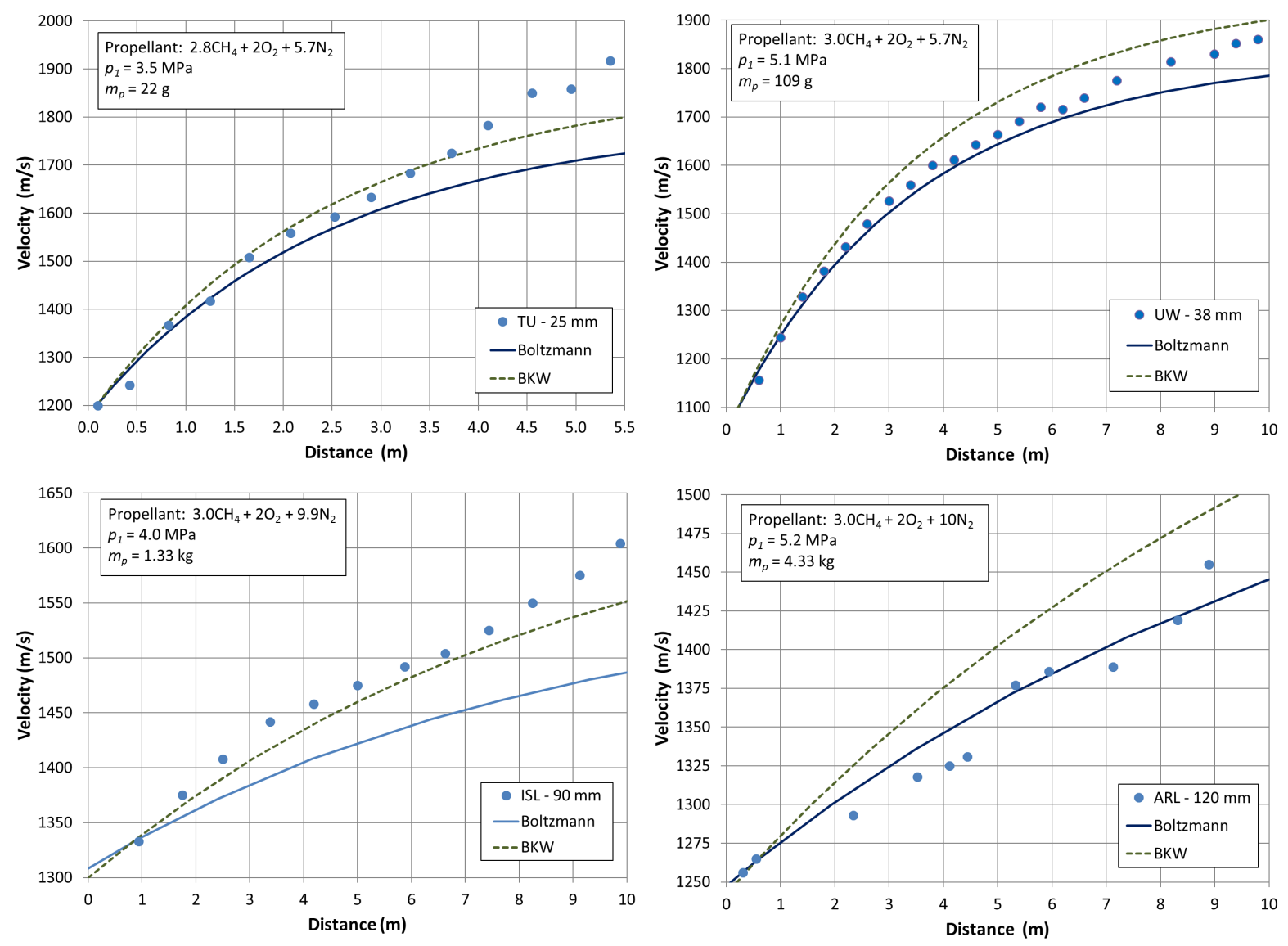

Figure 4. Experimental velocity-distance data compared with unsteady, 1-D theory using a constant length combustion zone $(350 \mathrm{~mm})$ and the Boltzmann and BKW EoS (experimental parameters in Table 1).

Note that the experimental velocity data begin to deviate upward from the theoretical predictions in all cases. This behaviour is attributed to the combustion process moving up on the projectile body and the cessation of thermal choking at full tube area behind the projectile as the projectile velocity approaches $D_{C J}$. An investigation of the factors leading to these thrust deviations from theory are beyond the scope of present work, thus experimental data at even higher velocities from these experiments than those shown are not plotted nor considered.

\subsection{Non-Dimensional Thrust}

Non-dimensional thrust vs. Mach number data from the experiments presented in Fig. 4 are plotted in Fig. 5. The scatter in the experimental acceleration data is much larger than with velocitydistance because most of it is derived by doubly differencing the original time-distance data. Nevertheless, the non-dimensional thrust calculations based on the BKW EoS are in better agreement with experimental results at fill pressures below $4 \mathrm{MPa}$ (TU and ISL) and the Boltzmann EoS results agree better when the fill pressure is greater than $5 \mathrm{MPa}$ (UW and ARL), which is consistent with the observations made in the velocity-distance data.

In Fig. 5, upsweeps in thrust are seen to begin at $M_{1} \sim 4.4,4.8,4.2$, and 3.8 in the experimental data from TU, UW, ISL, and ARL, respectively. These values are listed in Table 2 as "Deviation Mach" along with other parameters deduced from the experimental results and theoretical calculations. For the purposes of this study, we only consider the projectile to be operating in the thermally choked RAMAC propulsive mode from the initial $M_{l}$ data point to the Deviation Mach. The average acceleration is thus that determined for all the acceleration data between these two Mach numbers. The peak acceleration is determined by averaging the first two data points to smooth out the 

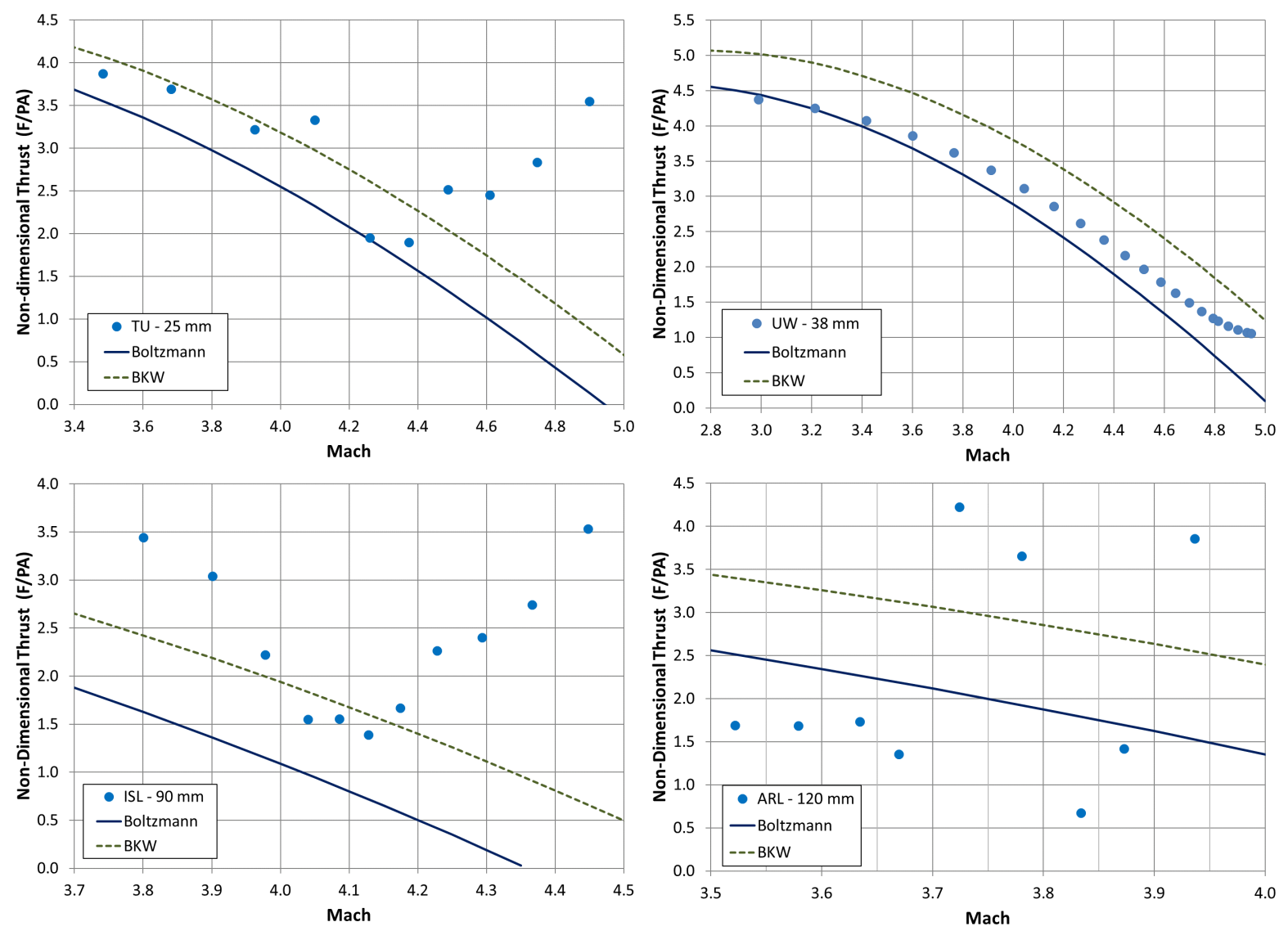

Figure 5. Experimental non-dimensional thrust vs. Mach number data from experiments shown in Fig. 4 compared with unsteady, 1-D theory using a constant length combustion zone $(350 \mathrm{~mm})$ and the Boltzmann and BKW EoS.

Table 2 Acceleration - Mach results for experiments from different RAMAC facilities.

\begin{tabular}{|c|c|c|c|c|c|c|c|c|}
\hline Facility & $\begin{array}{l}\text { Bore } \\
(\mathrm{mm})\end{array}$ & $\begin{array}{c}\text { Fill Pres } \\
\text { (MPa) }\end{array}$ & $\begin{array}{c}\text { End State } \\
\text { Pres (MPa) }\end{array}$ & $\begin{array}{l}\text { Mach } \\
\text { Range }\end{array}$ & $\begin{array}{l}\text { Deviation } \\
\text { Mach }\end{array}$ & $\begin{array}{c}\text { Avg Accel } \\
\left(\mathrm{km} / \mathrm{s}^{2}\right)\end{array}$ & $\begin{array}{c}\text { Peak Accel } \\
\left(\mathrm{km} / \mathrm{s}^{2}\right)\end{array}$ & $\begin{array}{l}\text { Projectile } \\
\text { Material }\end{array}$ \\
\hline TU & 25 & 3.5 & $31-42$ & $3.5-4.9$ & 4.4 & 230 & 290 & $\mathrm{Mg}$ \\
\hline UW & 38 & 5.1 & $37-70$ & $3.0-4.9$ & 4.8 & 150 & 230 & $\mathrm{Ti}$ \\
\hline ISL & 90 & 4.0 & $38-43$ & $3.8-4.5$ & 4.2 & 42 & 66 & $\mathrm{Al}$ \\
\hline ARL & 120 & 5.2 & $44-49$ & $3.5-4.0$ & 3.8 & 32 & 33 & $\mathrm{Al}$ \\
\hline
\end{tabular}

noise inherent in these experimental measurements. Note that only the TU and UW accelerations are in the range where unsteady effects are known to be significant, however, the unsteady modeling still gives an appropriate account of the EoS for the larger bore facilities because the unsteady effects diminish to zero as the projectile acceleration decreases.

The "end state pressure" column lists the pressure calculated at the thermal choking state for the first Mach number and the Deviation Mach data points, based on the EoS that best matched the experimental data. Note that these values range from 31 to $70 \mathrm{MPa}$ and are on the lower end of where real-gas effects are expected to be significant; i.e., $p_{2}>50 \mathrm{MPa}$. Nevertheless, the correlations of theoretical thrust based on these EoS are still of interest; particularly since ideal gas calculations under-predict the experimental results. The application of this modeling approach to experiments with higher fill pressure is warranted to better ascertain at which point should an EoS other than Boltzmann be used for thrust calculations.

The final column in Table 2 lists the projectile material for each experiment. The influence of projectile material on the thrust-Mach behaviour of the experiments has been found to be significant at 
elevated pressures and high Mach number [30,31]. In Fig. 5, it is evident that the larger bore facilities (ISL and ARL) experience an earlier onset of Deviation Mach when using Al-alloy projectiles than either of the smaller bore facilities. This may be due to the combustion zone length being smaller relative to length of projectile (i.e., $350 \mathrm{~mm}$ vs. $L_{p}=396$ or $522 \mathrm{~mm}$ ) which, due to the relatively large amount of surface area exposed to the flow, could promote projectile material combustion. It is also noteworthy that the 25-mm-bore TU facility experiences earlier onset of Deviation Mach with shorter residence times and shorter tube lengths than the 38-mm-bore UW facility using a Ti-alloy projectile. In these scenarios, it is evident that the Ti-alloy requires significantly more heat transfer than the Mgalloy before metallic combustion begins (if indeed this does occur in this situation). The utilization of ram accelerator projectile material combustion has been deliberately applied in the ISL facility [30], and is a wide open topic in need of deeper study in future.

\section{Conclusion}

Refinements to the one-dimensional model of the thermally choked ram accelerator propulsive mode include real-gas effects and unsteady assumptions to predict thrust vs. Mach number characteristics. Experiments from facilities having bore dimensions ranging from 25 to $120 \mathrm{~mm}$ were modelled. Comparisons of the computed thrust-Mach number profiles with experiments show that, among the equations of state considered, the Boltzmann EoS, despite its simple molecular interaction law, is appropriate for $5 \mathrm{MPa}$ propellant fill pressure in the $38-\mathrm{mm}$ and 120 -mm-bore facilities. Although it tends to over-predict the experimental data from the $38-\mathrm{mm}$-bore device, the empirical BKW EoS turns out to improve the theoretical agreement with experimental data from the $25-\mathrm{mm}$ and 90-mm-bore facilities where the fill pressures were less than $4 \mathrm{MPa}$. Calculations which include unsteady effects are generally in better agreement with experiments than those using a quasi-steady model, even when using a fixed-length for the combustion zone. Evidence of projectile material combustion was seen in that the larger Al-alloy projectiles tended to experience enhanced thrust at lower Mach than the smaller ones made from Mg and Ti-alloy. Although deviations of the calculated thrust vs. Mach number characteristics from experiment were evident under the circumstances considered here, the potential of the model to predict the thrust behaviour of the ram accelerator over a relative wide range of fill pressures and projectile size scales make it a useful tool for the first order parametric investigations.

\section{REFERENCES}

1. Hertzberg, A., Bruckner, A.P., and Bogdanoff, D.W., "Ram Accelerator: A New Chemical Method for Accelerating Projectiles to Ultrahigh Velocities," AIAA J., Vol. 26, No. 2, 1988, pp. 195-203.

2. Bruckner, A.P., Knowlen, C., Hertzberg, A., and Bogdanoff, D.W., "Operational Characteristics of the Thermally Choked Ram Accelerator" J. of Propulsion and Power, Vol. 7, No. 5, 1991, pp. 828-836.

3. Hertzberg, A., Bruckner, A.P., and Knowlen, C., "Experimental Investigation of Ram Accelerator Propulsion Modes," Shock Waves, Vol. 1, No. 1, 1991, pp. 17-25.

4. Bundy, C., Knowlen, C., and Bruckner, A.P., "Unsteady Effects on Ram Accelerator Operation at Elevated Fill Pressures," J. of Propulsion and Power, Vol. 20, 2004, pp. 801-810.

5. Giraud, M.J., Legendre, J.F., and Henner, M., "RAMAC in Subdetonative Propulsion Mode: State of the ISL Studies," Ram Accelerators, Takayama K., Sasoh A. (eds.), Springer-Verlag, Heidelberg, 1998, pp. 65-78.

6. Kruczynski, D.L. and Liberatore, F., "Experimental Investigation of High Pressure/Performance Ram Accelerator Operation," 1996, AIAA 96-2676.

7. Y. Hamate, A. Sasoh, and K. Takayama, "High Acceleration Using Open-Base Projectile," J. of Propulsion and Power, Vol. 19, No. 2, 2003, pp. 801-810.

8. Bengherbia, T., Yao, Y.F., and Bauer, P., "Computational Investigation of Transitional Viscous Flow over a Ram Accelerator Projectile in Sub-Detonative Propulsion Mode," 2006, AIAA 2006-0558.

9. Bengherbia, T., Yao, Y.F., Bauer, P., and Knowlen, C., "Numerical Investigation of Thermally Choked Ram Accelerator in Sub-Detonative Regime," 2009, AIAA 2009-0635. 
10. Bengherbia, T., Yao Y.F., Bauer P., and Knowlen, C., "CFD-based 1D Modeling of the Thermally Choked Ram Accelerator," The 50 th AIAA Aerospace Sciences Meeting and Exhibit, Nashville, TN, 2012, AIAA 2012-982.

11. Choi, J.-Y., Lee, B.-J. and Jeung, I.-S. "Computational modeling of high pressure combustion mechanism in scram accelerator," J. Phys. I V France 10, 2000, Pr11-131.

12. C. Li and K. Kailasanath, "Initiation Mechanism of the Thermally Choked Combustion in Ram Accelerators," J. of Propulsion and Power, Vol. 15, No. 1, 1999, pp. 151-153.

13. Nusca M.J. and Kruczynski, D.L, "Reacting Flow Simulation for Large-Scale RAMAC," $J$. of Propulsion and Power, Vol. 12, No. 1, 1996, pp. 61-69.

14. Bengherbia, T., Yao Y.F., Bauer P., and Knowlen, C., "One-dimensional Performance Modeling of the RAMAC in Subdetonative Regime," Aerotecnica, J. of Aerospace Sci., Tech. and Syst., Vol. 89, 2010, pp. 3-13.

15. Bengherbia, T., Yao Y.F., Bauer P., Giraud, M., and Knowlen, C., "Improved 1-D Unsteady Modeling of the Thermally Choked RAMAC in the Sub-Detonative Propulsion Mode," J. of Appl. Mech., Vol. 78, 2011, pp. 150-167.

16. P. Bauer, T. Bengherbia, C. Knowlen, A.P. Bruckner, Y. Yao, and M. Giraud, "Equations of State Implementation for 1-D Modelling of Performance in Ram Accelerator Thermally Choked Propulsion Mode," Int. J. Engineering systems Modelling and Simulations, Vol. 7, No. 2, 2015, pp 71-79.

17. Bauer, P., Knowlen, C., and Bruckner, A.P., "Real gas effects on the prediction of ram accelerator performance," Shock Waves, Vol. 8, 1998, pp. 113-118.

18. Bauer, P. and Knowlen, C., "Compressibility effects of unreacted propellant on thermally choked ram accelerator performance," Eur. Phys. J. Appl. Phys., Vol. 21, 2003, pp.233-238.

19. Bauer, P., Knowlen, C., and Bruckner, A.P., "Modeling Acceleration effects on Ram Accelerator Thrust at High Pressures," J. of Propulsion and Power, Vol. 21, 2005, pp. 955-957.

20. K.W. Naumann and A.P. Bruckner, "Ram Accelerator Ballistic Range Concept for Softly Accelerating Hypersonic Free-Flying Models," J. of Aircraft, Vol. 31, No. 6, 1994, pp 1310-1316.

21. C. Knowlen and A.P. Bruckner, "Direct Space Launch Using Ram Accelerator Technology," Space Technology and Applications International Forum-2001, ed M.S. El-Genk, American Institute of Physics, 2001, pp 583-588.

22. Heuzé, O., "Equations of State of Detonation Product," Phys. Rev. A, Vol. 34, 1986, pp. 428-432.

23. D.L. Buckwalter, C. Knowlen and A.P. Bruckner, "Real Gas Effects on Ram Accelerator Analysis," 1997, AIAA 97-2894.

24. Bauer, P., Brochet, C., and Krishnan S., "Detonation Characteristics of Gaseous Ethylene, Oxygen and Nitrogen Mixtures at High Initial Pressures," Progress in Aeronautics and Astronautics, Ed. AIAA, New York, NY, Vol. 75, 1981, pp. 408-422.

25. Bauer, P., Presles, H.N., Heuzé, O., and Brochet, C., "Equation of State for Dense Gases," Archivum Combustionis, 5, 1, 1985, pp. 315-320.

26. Mader, C.L., "Detonation Properties of Condensed Explosives Computed Using the BeckerKistiakowsky-Wilson Equation of State," Report-LA-2900, 1963, Los Alamos Scientific Laboratory, NM, USA.

27. Bauer, P., Dunand, M., and Presles, H.N., "Detonation Characteristics of Gaseous Methane-OxygenNitrogen Mixtures at Extremely Elevated Initial Pressure," AIAA Progress in Aeronautics and Astronautics, Vol. 133, 1991, pp. 56-62.

28. Bauer, P., Legendre, J.F., Knowlen, C., and Higgins, A.J., "Transition of Detonation on Insensitive Dense Gaseous Mixtures in Tubes," 1996, AIAA 96-2682.

29. Bengherbia, T., Yao Y.F., Bauer P., and Knowlen, C., "Thrust Prediction in Thermally Choked Ram Accelerator," 2010, AIAA 2010-1129.

30. J.-F. Legendre and M. Giraud, "Enhanced RAMAC performance in the Subdetonative Propulsion Mode with Semi-Combustible Projectile," J. Phys. IV France, Vol. 10, 2000, Pr11-23-Pr11-30.

31. A.J. Higgins, C. Knowlen and A.P. Bruckner, "Ram Accelerator Operating Limits, Part 2: Nature of Observed Limits," J. of Propulsion and Power, Vol. 14, No. 6, 1998, pp. 959-966. 